\title{
BMJ Open Study protocol for a phase II, multicentre, prospective, non- randomised clinical trial to assess the safety and efficacy of infusing allogeneic activated and expanded natural killer cells as consolidation therapy for paediatric acute myeloblastic leukaemia
}

To cite: Muñoz Builes M, Vela Cuenca M, Fuster Soler JL, et al. Study protocol for a phase II, multicentre, prospective, non-randomised clinical trial to assess the safety and efficacy of infusing allogeneic activated and expanded natural killer cells as consolidation therapy for paediatric acute myeloblastic leukaemia. BMJ Open 2020;10:e029642. doi:10.1136/ bmjopen-2019-029642

- Prepublication history and additional material for this paper are available online. To view these files, please visit the journal online (http://dx.doi. org/10.1136/bmjopen-2019029642).

Received 14 February 2019 Revised 14 December 2019 Accepted 17 December 2019

\section{Check for updates}

(C) Author(s) (or their employer(s)) 2020. Re-use permitted under CC BY-NC. No commercial re-use. See rights and permissions. Published by BMJ.

For numbered affiliations see end of article.

\section{Correspondence to} Dr Antonio Pérez-Martínez; aperezmartinez@salud.madrid. org

\section{Mario Muñoz Builes (D) , ${ }^{1}$ María Vela Cuenca, ${ }^{2}$ Jose L Fuster Soler, ${ }^{3}$ Itziar Astigarraga, ${ }^{4}$ Antonia Pascual Martínez, ${ }^{5}$ Jose M Vagace Valero, ${ }^{6}$ Hoi Y Tong, ${ }^{1}$ Jaime Valentín Quiroga, ${ }^{2}$ Lucía Fernández Casanova, ${ }^{7}$ Adela Escudero López, ${ }^{8}$ Luisa Sisinni, ${ }^{9}$ Miguel Blanquer (1) , ${ }^{3}$ Isabel Mirones Aguilar, ${ }^{2}$ Berta González Martínez, ${ }^{2,9}$ Alberto M Borobia, ${ }^{10}$ Antonio Pérez-Martínez ${ }^{2,9}$}

\section{ABSTRACT}

Introduction Acute myeloblastic leukaemia (AML) constitutes the second most common haematological malignancy in the paediatric population. Current treatment regimens are based on the administration of polychemotherapy, combining high doses of cytarabine with anthracyclines and topoisomerase inhibitors. Allogeneic haematopoietic stem cell transplantation (HSCT) is an option for high-risk patients with AML (and for intermediate-risk patients if a sibling donor is available). With this strategy, AML survival has increased substantially; however, it has remained stagnant at approximately $60 \%$, with relapse being the principal culprit. The predominant role of the immune system and natural killer (NK) cells in controlling paediatric AML has gained importance within the context of HSCT. In this protocol, we propose incorporating this cell therapy as an adjuvant treatment through the infusion of activated and expanded haploidentical NK (NKAE) cells in paediatric patients with AML who are in cytological remission after completing consolidation therapy, and with no indication for HSCT.

Methods and analysis Patients up to 30 years of age, diagnosed with AML, in their first cytological remission, who have completed both the induction and the consolidation phases of chemotherapy and do not meet the criteria for allogeneic HSCT are eligible. The patients will receive two doses of NKAE cells once a week, using a GMP K562-mbIL15-41BBL stimulus from a haploidentical donor and interleukin 2 subcutaneously. The patients will then be followed up for 36 months to assess the primary endpoint, which is the probability of relapse after NK cell infusion.

\section{Strengths and limitations of this study}

- Prospective phase II clinical trial with up to six paediatric institutions participating.

- The anti-leukaemia effect has been previously described in patients with acute myeloblastic leukaemia, mediated by alloreactive natural killer (NK) cells.

- It is not a randomised clinical study.

- The anti-leukaemia effect of activated and expanded NK cells needs to be evaluated in combination with chemotherapy.

Ethics and dissemination This clinical trial was approved by the Clinical Research Ethics Committee of La Paz University Hospital and The Spanish Agency of Medicines and Medical Devices. Findings will be disseminated through peer-reviewed publications, conference presentations and community reporting. Trial registration number EudraCT code: 2015-00190115, ClinicalTrials.gov Identifier: NCT02763475.

\section{INTRODUCTION}

Acute myeloblastic leukaemia (AML) is the most common haematological malignancy in adults and the second most common haematological cancer in children and adolescents. ${ }^{1}$ The incidence rate is approximately seven cases per million children per year in the paediatric population, and accounts for $5 \%$ of 
all paediatric cancers and $20 \%$ of all paediatric leukaemia. The 5-year survival rate is approximately $62 \%$ (95\% CI $54 \%$ to $69 \%$ ) according to data from the Spanish Registry of Childhood Tumours, still far from the current survival rate for paediatric acute lymphoblastic leukaemia. Therefore, new therapeutic strategies are needed. ${ }^{2}$ Molecular cytogenetics and molecular biology have managed to identify genetic lesions, which have helped deepen our understanding of AML, and has classified them according to morphological, clinical and molecular criteria, relating these to prognoses. ${ }^{3}$ Recent comparative genomic hybridisation studies have highlighted the heterogeneity of AML, revealing new genetic subtypes, identifying prognosisrelated genetic lesions and, above all, opening the door to the development of target drugs. Except for the AML promyelocytic subtype (AML M3), targeted therapies to date have failed in various clinical trials, highlighting the need for new approaches. ${ }^{4}$ Our understanding of these new pathophysiological mechanisms and the failure of target drugs are enabling the current development of AML immunobiology, using adoptive cell therapy. ${ }^{5}$ Clinical and preclinical studies have revealed the important role of the immune system in controlling AML. Myeloid leukaemia cells acquire properties of immortalisation, growth, resistance to apoptosis and changes regarding the bone marrow microenvironment. The immune system can destroy these leukaemia cells and can also select the less immunogenic ones, in a complex immunoediting process. The loss of interaction between leukaemia cells and the immune system promotes leukaemic progression and helps the cells evade immune system control. ${ }^{6}$

The anti-leukaemia effect of immune cells was first described in murine models in the 1970s, in which leukaemic blast cells were eliminated by allogeneic mononuclear cells but were not eliminated by syngeneic mononuclear cells. This anti-leukaemia effect is mediated primarily by the $\mathrm{CD} 8^{+}$and $\mathrm{CD} 4^{+} \mathrm{T}$ lymphocyte subsets, which recognise minor antigens (such as LRH1) and leukaemia-associated antigens (BCR/ABL, PML/ RARa, WT1, PR3) on the surface of leukaemic blasts in human leucocyte antigen (HLA)-matched allogeneic haematopoietic stem cell transplantation (HSCT). ${ }^{7}$ Determining the role of natural killer (NK) cells and their anti-leukaemia effect is perhaps one of the greatest scientific milestones of the past decade and has helped develop cell therapy platforms based precisely on the HLA differences between donor and recipient. ${ }^{8}{ }^{9}$ Allogeneic NK cells that express inhibitory receptors, such as killer immunoglobulin-like receptors (KIRs), and that are not inhibited by the HLA molecules of the patient's leukaemic blast cells cause a powerful anti-leukaemia effect (KIR-HLA mismatch). This effect has been developed in the clinical context of HLA mismatch HSCT, and it has been primarily successful in preventing relapse in patients with AML, in both adults and children. ${ }^{10}$ NK cells are ideal candidates for cancer immunotherapy and meet a number of criteria: (1) speed of action; (2) potency; (3) broad specificity; (4) potential for selecting optimal donors; (5) ease of preparation and administration; (6) safety profile with lack of serious adverse effects; and (7) complementary nature with other therapeutic approaches, such as chemotherapy. NK cell infusion is an emerging therapy, which is increasingly gaining strength due to the solid available scientific evidence showing both its efficacy and safety.

The anti-tumour activity of NK cells in animals has been known for years; however, its transfer to humans is much more recent. Adoptive immunotherapy with NK cells for cancer has been developed in parallel with the development of biotechnology, coinciding with good manufacturing practice (GMP) platforms in various clinical contexts. ${ }^{11} 12$ See online supplementary data table S1 shows the clinical trials registered worldwide of cancer immunotherapy using NK cells.

In terms of therapeutic efficacy, one of the most relevant clinical trials has been implemented for paediatric AML by researchers at St. Jude's Children's Research Hospital, Memphis, Tennessee, USA. The preliminary results of this pilot study, with more than 2 years of follow-up, have been highly encouraging. None of the low-risk to intermediaterisk paediatric patients with AML who underwent consolidation therapy with NK cells obtained by apheresis from haploidentical donors have experienced a relapse in more than 2 years of follow-up. This result suggests a powerful anti-leukaemia effect of NK cells through the interaction of KIRs with myeloid blast cells without their respective ligands. The cell therapy strategy using NK cells as consolidation could be effective in conditions of minimal disease, eliminating those residual blast cells that are resistant to chemotherapy and that are responsible for leukaemia relapses. ${ }^{5}$ Due to patients' NK cell cytotoxicity impairment, the anti-leukaemic effect of allogeneic NK cell infusion could interact with the ligands for inhibitory ${ }^{13} 14$ and activating receptors, such as NKG2D. ${ }^{15}$ However, the adoptive transfer of NK cells did not reduce the cumulative incidence of relapse or overall survival over chemotherapy alone in a recent phase II study. ${ }^{16}$

Currently, the main obstacle to the clinical use of NK cells is their low number in peripheral blood and the need to work in conditions of human clinical use and with GMPs. However, using clinical-scale immunomagnetic separation with leukapheresis-based Macs biotechnology and/or the expansion of peripheral blood in GMP conditions, millions of NK cells with a purity greater than $95 \%$ can be infused. ${ }^{17} 18$ Interleukin 2 (IL-2) increases the activity of these cells after 24 hours of incubation and increases NK cell proliferation. Other cytokines, such as IL-4, IL-7 and IL-12, also induce NK cell proliferation but with less potency than IL-2. ${ }^{19}$

The group led by professor Darío Campana at St. Jude's Hospital in Memphis, Tennessee, USA, had used the genetically modified K562 cell line as an NK cell stimulant. ${ }^{2021}$ This cell line results from the myeloblastic crisis of a patient with BCR-ABL1 chronic myeloid leukaemia. K562 is characterised by lack of KIR ligands and plenty of NKG2D ligands. K562 stimulates NK cells through 
NK activating receptors such as NKG2D. The St. Jude group has developed this cell line for human use, with genetic changes that include the expression of two stimulatory molecules for the activating receptors of NK cells. One of these molecules is the ligand for receptor 4-1BB (CD137), and the other is IL-15, an essential cytokine for the cellular homeostasis of NK cells. ${ }^{22}$ Complete activation of NK cells is thereby achieved through three routes: 'missing-self,' through inhibitory KIR receptors; 'induced-self,' through the activating receptor (CD137) and 'cytokine-driven activation' with IL-15. The resulting cell line is called K562-mb15-41BBL and provides a powerful stimulus for the proliferation and activation of NK cells. This cell culture technique is currently available in Spain and is being employed by academic researchers in our group in the context of three clinical trials with autologous NK cells for high-risk multiple myeloma and with allogeneic NK cells in acute refractory leukaemia in children, adolescents and young adults. ${ }^{17} 1823$

In our experience, the 5-year survival rate of AML is approximately $61 \%$ (95\% CI $55 \%$ to $67 \%$ ), with a likelihood of relapse of approximately $38 \%$ (95\% CI $31 \%$ to $48 \%){ }^{24}$ Event-free AML survival in the paediatric population has not changed substantially with the various protocols. However, one patient group considered to be at 'intermediate risk' has been identified because the group lacks the cytogenetic abnormalities that characterise those at high risk or those at favourable risk. These 'intermediate-risk' patients currently have no indication for HSCT in the first full remission unless they have an HLA-identical sibling. Disease-free survival, however, is approximately $53 \%$ (95\% CI $45 \%$ to $61 \%){ }^{24}$ In this patient group, the therapy intensification strategy using high doses of chemotherapy and autologous haematopoietic progenitor cell rescue has not improved survival. We therefore consider these patients to be ideal candidates for developing new therapeutic strategies such as NK cell therapy, under conditions of full remission, in which adoptive immunotherapy can be more effective and lacks significant complications.

In this clinical trial, we propose combining chemotherapy with activated and expanded natural killer (NKAE) cell infusion based on the synergistic anti-tumour effect of chemoimmunotherapy. The role of chemotherapy in this regimen is as follows: (1) to cause stress in the residual tumour cells, increasing the expression of NK cell ligands for the activating receptors; (2) immunosuppression in the patient, enabling immunotherapy with allogeneic NK cells from a haploidentical donor; and (3) the autologous recovery of NK cells, leading to immune reconstitution after chemotherapy, thereby continuing the anti-tumour effect in the absence of tumour inhibitory signals.

\section{METHODS AND ANALYSIS \\ Main objective}

The main aim of this study is to decrease the probability of relapse at 3 years by $20 \%$ for paediatric patients with AML during their first cytological remission, with no high-risk cytogenetic abnormalities, a good prognosis and no indication for allogeneic HSCT.

\section{Secondary objectives}

1. To determine the in vitro susceptibility of myeloid blast cells to allogeneic NKAEs.

2. To determine the safety of NKAE infusion along with the chemotherapy regimen.

\section{TYPE AND DESIGN OF THE CLINICAL TRIAL Study design}

This is a phase II, open, prospective, non-randomised, multicentre clinical trial to assess safety and efficacy.

\section{Patient selection}

The patients will be recruited at La Paz University Hospital and Hospital 12 de Octubre (Madrid, Spain), Maternal Paediatric Hospital of Badajoz (Extremadura, Spain), Cruces University Hospital (Barakaldo, Spain), Hospital Carlos Haya (Malaga, Spain) and Hospital Virgen de la Arrixaca (Murcia, Spain). The same inclusion and exclusion criteria will be applied at all centres.

\section{Inclusion criteria}

1. Patients between the ages of 0 and 30 years, diagnosed with AML, in their first cytological remission, who have completed the induction and consolidation phases of chemotherapy and who do not meet the criteria for allogeneic HSCT.

2. Karnofsky ( $<16$ years of age) /Lansky ( $\geq 16$ years of age) index $>60 \%$.

3. Mild to moderate $(<4)$ functional organ impairment (hepatic, renal, respiratory), according to the criteria of the National Cancer Institute (NCI CTCAE V.4).

4. Left ventricular ejection fraction $>39 \%$.

5. Adult patients who have voluntarily signed the informed consent document before the first intervention of the study, or minors whose representative or legal guardian has voluntarily signed the informed consent document before the first study intervention.

6 . For mature minors (12-17 years of age), their consent will be obtained, along with their legal guardian's signed consent.

7. Females of childbearing age should have a negative pregnancy test at the time of inclusion and should have access to highly effective contraceptive methods during their study participation and for the 30 days following the last visit. Male patients with reproductive capability must commit to the use of an adequate contraceptive barrier method during the study and up to 6 months afterwards.

8. Presence of a haploidentical donor.

\section{Exclusion criteria}

The patients MUST NOT meet any of the criteria listed below to participate in the study:

1. History of poor therapeutic compliance. 
2. Children with acute myeloid leukaemia of Down syndrome, juvenile myelomonocytic leukaemia or acute promyelocytic leukaemia.

3. Patients who, after a psychosocial assessment, are deemed unsuitable for the procedure.

- Social/family situation that precludes proper participation in the study.

- Patients with emotional or psychological problems secondary to the disease, such as post-traumatic stress disorder, phobias, delirium or psychosis, which require specialised support.

- Assessment of relatives' involvement in the patient's health.

- Inability to understand the trial information.

4. Severe functional impairment of organs (hepatic, renal, respiratory), according to the criteria of the $\mathrm{Na}-$ tional Cancer Institute (NCI CTCAE V.4.3).

5. Patients who have been administered other study drugs in the 90 days before inclusion.

\section{STUDY TREATMENT}

We propose infusing allogeneic NKAE cells from a haploidentical donor after administering lymphodepleting chemotherapy (cyclophosphamide $60 \mathrm{mg}$ / $\mathrm{kg}$ once and fludarabine $25 \mathrm{mg} / \mathrm{m}^{2} /$ day for 5 days) as consolidation therapy for patients with AML who have completed therapy and who are in cytological remission. The objective of the prior chemotherapy administration is to decrease effector $\mathrm{T}$ cells that would otherwise reject the infused donor NKAE cells. In conjunction with the infusion of NKAE cells, we will administer low doses $\left(1 \times 10^{6} / \mathrm{IU} / \mathrm{m}^{2}\right)$ of IL-2 subcutaneously every 48 hours for 2 weeks to promote the expansion and anti-leukaemia effect of the NKAE cells (figure 1).

\section{Selection of the donor}

Donors will be evaluated in the haematology department of the participating centres, as is common with donors for blood product transfusion procedures, cell therapy and HSCT. Only donors older than 18 years who are not pregnant or breast feeding will be accepted. The donor will be informed that if the investigators plan on new analyses related to the disease and/or to NK cells in the future, these would need approval by an accredited clinical research ethics committee, and the donor would be asked to sign a new informed consent document.

\section{Preservation of peripheral blood samples}

The peripheral blood samples will be preserved and stored in accordance with the current legislation, Royal Decree-Law 9/2014 of July 4, establishing the quality and safety standards for the donation, procurement, evaluation, processing, preservation, storage and distribution of human cells and tissues, and approving the coordination and operation standards for their use in humans.

\section{Manufacture of NKAE cells}

A total of $250-400 \mathrm{~mL}$ of peripheral blood from the haploidentical donor (father, mother or sibling) will be sent to the GMP-certified cell culture laboratory at University Hospital Niño Jesús, Madrid, Spain. The NKAE cell manufacturing will be performed following GMP standards in the Cell Therapy Unit of Hospital Niño Jesus (GMP accreditation since April 2010: UPTCHNJCertificate No. ES/158/13). There, a peripheral blood mononuclear cell (PBMC) culture will be performed, obtained through a Ficoll gradient, with the sublethally irradiated GMP-grade cell line K562-mb-IL15-4BBI. A PBMC/K562-mbIL-15-4BBI ratio of $1 / 1.5$ will be established, and after 2 weeks of culture in the Roswell Park Memorial Institute (RMPI) GlutaMAX medium 10\% human serum $+100 \mathrm{IU} / \mathrm{mL}$ IL-2, NKAE cells will be obtained. The expected NKAE cell ratio has been previously described. ${ }^{17}$ After the second week, up to $5 \times 10^{7} /$ $\mathrm{kg}$ of $\mathrm{CD} 56^{+} \mathrm{CD} 3^{-}$cells and ideally fewer than $1 \times 10^{6} / \mathrm{kg}$ of $\mathrm{CD}^{\circ} 6^{-\mathrm{CD}^{+}}{ }^{+}$cells will be infused. However, residual T cells will not be removed. For cases in which the expansion fails or there is a considerable discrepancy between the weight of the donor and recipient or other medical criteria (eg, the possibility of shorter infusion time given the patient's clinical characteristics), NK cells may be obtained using immunomagnetic methods (CliniMacs) based on nonmobilised leukapheresis. This manufacturing method has been validated and submitted to the Spanish Agency for Medicinal Products and Medical Devices. Manufacturing approval was obtained for the Investigative Medicinal Product with code PEI-12-009.

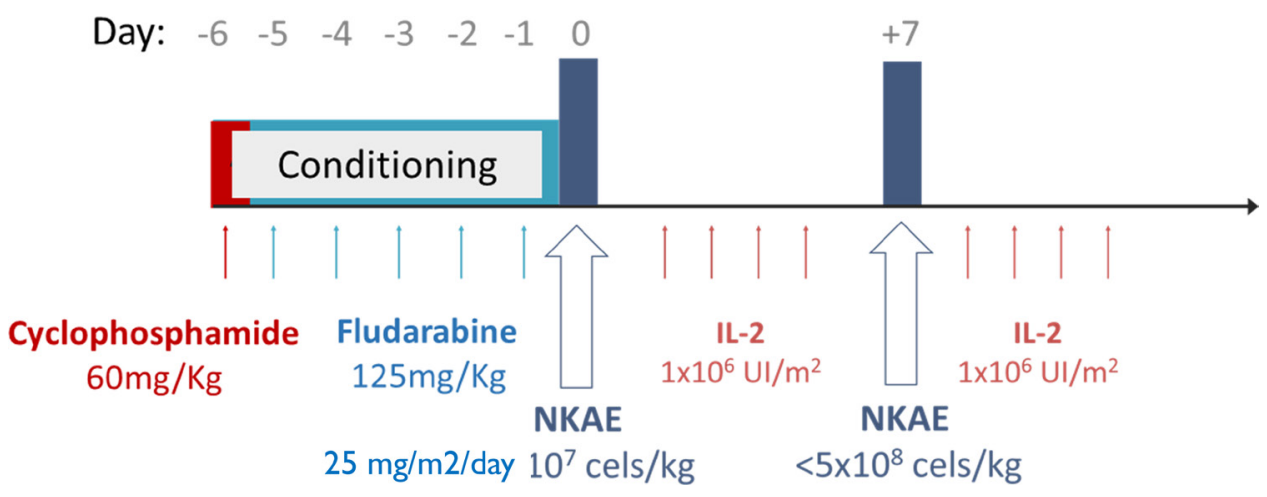

Figure 1 Treatment schedule. NKAE, activated and expanded natural killer cell. 


\section{ENDPOINTS}

\section{Primary endpoint}

Each patient will be monitored according to the clinical guidelines for detecting relapses. For their assessment, a bone marrow aspiration will be performed 1 month after completing the treatment and at least once a year thereafter until 3 years of follow-up have been completed. The objective response rate will be established according to cytomorphological criteria and by minimal residual disease (MRD) by cytometry and/or real-time PCR.

Relapse will be defined as the presence of myeloid blast cells with the same markers as found during diagnosis or with variations, and presenting the same or new molecular cytogenetic abnormalities. Relapse in bone marrow will be determined at the cytological level by the reappearance of leukemic blasts greater than $5 \%$ or by MRD by cytometry $(>0.01 \%)$ and/or molecular methods $(0.0001 \%)$ present at diagnosis with or without cytogenetic abnormalities.

\section{Secondary endpoints}

1. Safety of NKAE cell infusion along with the chemotherapy regimen.

2. HLA typing of patient and donor, KIR haplotype assessment of donor and NK functionality of donor against patient's leukaemia.

3. Haematopoietic chimerism after NKAE cell infusion.

4. Ligand expression on leukemic blasts at diagnosis, or relapse for the NKG2D activating receptor (MICA, MICB, ULBP) and KIR inhibitory receptors (HLA-I) will be determined by multiparametric flow cytometry.

5. The in vitro cytotoxic activity of the allogeneic NKAE cell versus the leukaemic blast cells will be another variable measured with real-time Eur-TDA fluorescence.

\section{CLINICAL TRIAL ASSESSMENT AND CLINICAL FOLLOW-UP}

The clinical trial assessment is summarised in table 1 .

\section{Inclusion phase (visit 1)}

The participants will be enrolled from the paediatric haematology oncology department of the six hospitals participating in the study. The enrolment phase will last 32 months.

Verbal and written information about the study will be provided to the patient. The patient/family member/ guardian must grant their consent in writing and in duplicate to participate in the clinical trial. The maximum period from the signing of the informed consent to the start of the chemotherapy treatment phase will be 30 days. Visit 1 can be completed in more than one visit according to the patient's or researcher's requirements. The researchers will check and record in the case report form (CRF) whether the patient meets all inclusion criteria and none of the exclusion criteria. Demographic data, personal medical history and a physical examination will be recorded in the CRF. A blood test, leucocyte subpopulation study from peripheral blood samples, urinalysis, echocardiogram and bone marrow aspirate will also be performed.

\section{Treatment phase (visits 2 to 15): chemotherapy} administration, NKAE cells and IL-2

At each visit, a physical examination will be performed and vital signs will be recorded, as well as any concomitant medications and adverse events. On completing conventional AML therapy, and with haematological recovery, a chemotherapy cycle will be scheduled:

- Visit 2 (day -6): $60 \mathrm{mg} / \mathrm{kg}$ cyclophosphamide intravenously.

- Visit $3($ day -5$)$, visit $4($ day -4$)$, visit 5 (day -3$)$, visit 6 (day -2 ) and visit 7 (day -1$): 25 \mathrm{mg} / \mathrm{m}^{2} /$ day fludarabine intravenously.

- Visit 8 (day 0): NKAE cell infusion will be administered 24 hours after completing chemotherapy. During this first infusion, $5 \times 10^{7}$ cells $/ \mathrm{kg}$ with immunophenotype NK $\left(\mathrm{CD}^{-} \mathrm{CD} 56^{+}\right)$will be infused. A few hours before the infusion, IL-2 $\left(1 \times 10^{6} \mathrm{IU} / \mathrm{m}^{2}\right)$ will be administered subcutaneously.

- Visit 9 (day +2): IL-2 $\left(1 \times 10^{6} \mathrm{IU} / \mathrm{m}^{2}\right)$ will be administered subcutaneously. A $10 \mathrm{~mL}$ blood EDTA tube will be sent to La Paz University Hospital, Madrid, Spain, to study NK cell phenotype and chimerism.

- Visit $10($ day +4$)$ and visit $11($ day +6$):$ IL-2 $\left(1 \times 10^{6} \mathrm{IU} /\right.$ $\mathrm{m}^{2}$ ) will be administered subcutaneously.

- Visit $12($ day +7$)$ : the second NKAE cell infusion will be administered 1 week after the first administration. In this second infusion, up to $5 \times 10^{8}$ cells $/ \mathrm{kg}$ will be infused, provided there has been no toxicity attributable to the infusion of the cell product in the previous cycle. As the previous infusion, ideally fewer than $1 \times 10^{6} / \mathrm{kg}$ of $\mathrm{CD} 56 \mathrm{CD}^{+}$cells will be infused. However, residual $\mathrm{T}$ cells will not be removed.

- A $10 \mathrm{~mL}$ blood EDTA tube will be sent to La Paz University Hospital, Madrid, Spain, to study NK cell phenotype and chimerism.

- Visit $13($ day +8$)$ and visit $14($ day +10$):$ IL-2 $\left(1 \times 10^{6}\right.$ IU/ $\mathrm{m}^{2}$ ) will be administered subcutaneously.

- Visit 15 (day +15): a $10 \mathrm{~mL}$ blood EDTA tube will be sent to La Paz University Hospital, Madrid, Spain, to study NK cell phenotype and chimerism.

Before administering the corresponding drug, a physical examination will be performed, and any adverse events and changes in concomitant medication will be recorded.

\section{Follow-up phase (visits 16 to 26)}

Patients will have a 36-month follow-up from the end of therapy. During this phase, the assessment of the study's primary endpoint (the onset of a relapse) will be evaluated using bone marrow aspirate. Likewise, all adverse events that arise, including those related to the extraction of samples, the NKAE cell administration procedure, immunological abnormalities, allergic reactions and the onset of potential secondary malignancies will be documented during this phase. 


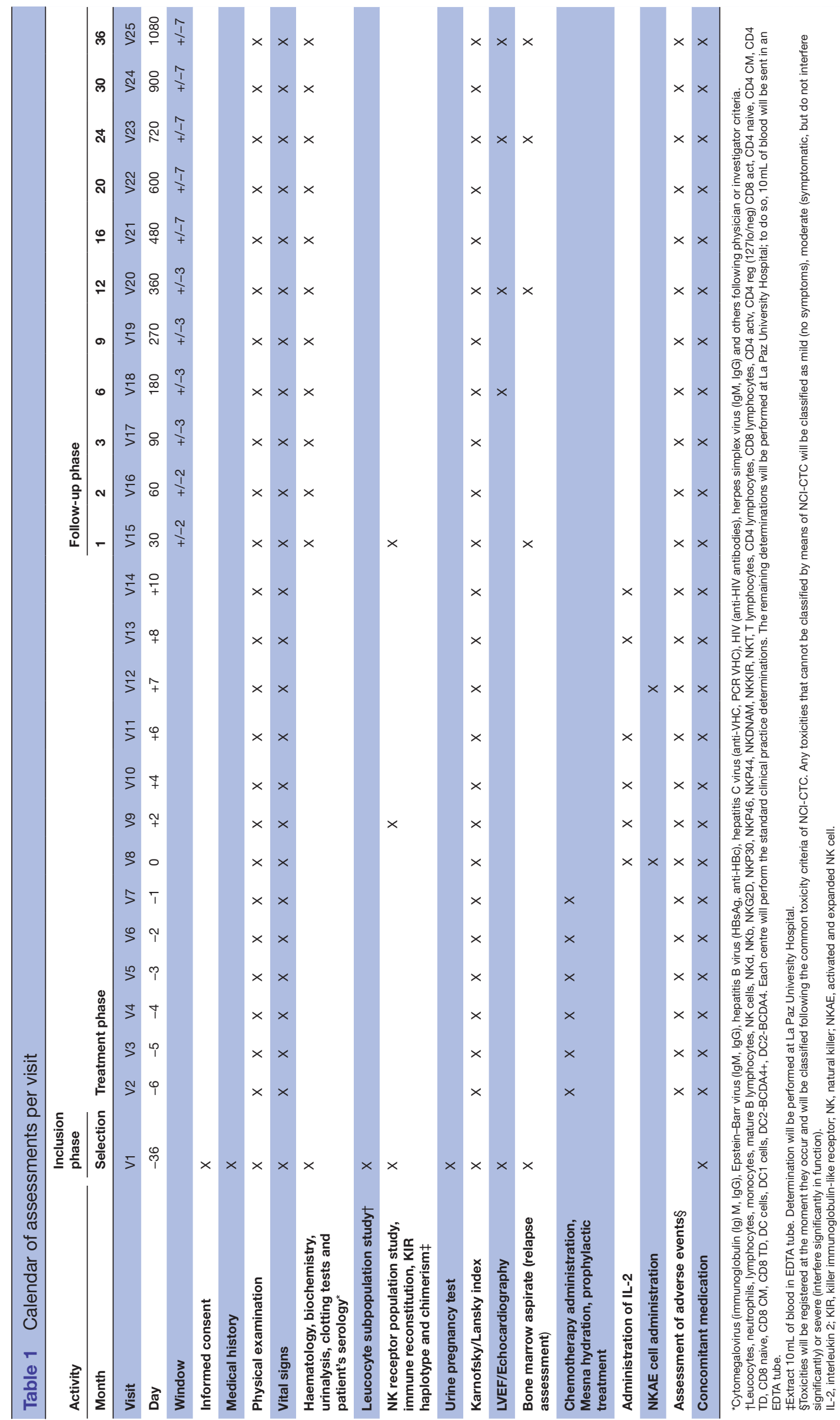


The visits will be conducted as follows:

- V16 to V26: during all visits, a physical examination will be performed, including laboratory tests for blood counts, biochemistry, coagulation, Karnofsky/Lansky index, evaluation of adverse events and changes in concomitant medication.

- In addition, at V16, V21 and V26, a bone marrow aspiration will be performed to assess relapse (eg, complete response, partial response, disease progression, stable disease, MRD) since the first NKAE cell administration.

- V19, V21, V24 and V26 will also include an echocardiogram.

End of study: The end of the study will be the day of the last visit of the last patient.

\section{ADVERSE EVENTS}

Adverse events should be identified and reported promptly, in such a way that the potential patient risks can be determined, and in compliance with regulatory requirements. To assess adverse events, we will follow NCI-CTC V4.0 criteria and will determine the proportion of patients who have toxicity according to degree.

\section{DATA MONITORING}

Coordination, management and monitoring of the study will be performed by the Spanish Clinical Research Network.

\section{STATISTICAL ANALYSIS}

\section{Sample size}

We expect to recruit 35 clinically evaluable patients for the objective of efficacy. The sample size of 35 patients has been calculated considering that the 3-year relapse rate in children and adolescents with AML is approximately $40 \%$. We believe that cell therapy using an infusion of NKAE cells from a haploidentical donor can reduce the relapse rate at 3 years by up to $20 \%$. To detect this reduction, with a power of $80 \%$ and assuming an alpha error of 0.05 , we need to include a total of 35 patients.

\section{Statistical analysis}

The statistical tests and the specific study of each endpoint will be reported in detail in the Statistical Analysis Plan, which will be performed before closing the database. Nevertheless, a brief description of these tests and the study is given next. The analysis will be performed with the SPSS statistical package, V.15.0 or later. For the description of the continuous endpoints, we will use the mean, SD, median and range. When necessary, we will calculate $95 \%$ CIs. We will provide the objective response rate and its corresponding 95\% CI. A survival analysis (Cox regression) will be performed to assess the time from undergoing NKAE cell treatment to the onset of recurrence (if there is one).
The safety profile will be reported using the NCI-CTCV4.0 classification, and the number and percentage of patients who present various adverse events will be provided, indicating the severity and its association with the treatment.

All cases in which the treatment was suspended due to an adverse event will be reported, along with all deaths due to toxicity.

\section{PATIENT AND PUBLIC INVOLVEMENT}

The development of the research question and outcome measures were based on the oncologists' experience treating patients with AML and the desire to find better treatment options. Patients and patient advisers were not involved in the design, recruitment or conduct of this study. The patients or their families will be notified of the study results in writing and verbally.

\section{ETHICS AND DISSEMINATION}

This study will be conducted using the principles established in the latest version of the Declaration of Helsinki (Fortaleza, Brazil, October 2013) and current Spanish legislation on clinical trials. The study will be performed according to good clinical practice standards/International Conference on Harmonisation. This study has been approved by the Clinical Research Ethics Committee of La Paz University Hospital, Madrid, Spain, and by the Spanish Agency of Medication and Health Products, and it has been registered in Eudra CT (2015-001901-15). An insurance policy has been contracted for the clinical trial. Outputs from this study will include journal publications, conference presentations and community reporting. Outputs will not identify participants.

\section{DISCUSSION}

The immune system constitutes the main 'therapy' for preventing cancer growth in most people who never develop cancer throughout their lifetime. The immune system is the only physiological (and, at the same time, a tremendously effective) tool in the healthy population. Understanding how healthy individuals continuously win the battle against cancer with only the assistance of their immune system can be used to develop therapies for patients who develop cancer. In this respect, immune and cell therapy are new therapeutic approaches. Cell therapy is an advanced and complex therapeutic modality, and any progress in its use is of considerable scientific relevance. There is currently considerable dissociation between conventional treatments (chemotherapy, radiation therapy and surgery) and the new immunological and cellular therapies, such as cell therapy that is reserved for highly advanced disease stages. In our opinion, immunological and cellular therapies should be combined with conventional treatments. Incorporating therapy with autologous and allogeneic NK cells constitutes a new and successful therapeutic approach in the leading clinical 
research centres. Previous data obtained with adoptive haploidentical KIR-HLA-mismatched NK cells offer safety, transient engraftment and efficacy in adult patients with AML. ${ }^{25}{ }^{26}$ Given the absence of adverse effects, repeated NK cell infusions should be possible to incorporate into a chemotherapy regimen during the induction or consolidation phases. In our trial, we propose developing a chemotherapy and immunotherapy regimen with NKAE cell that directly reduces the likelihood of relapse in patients with AML.

\section{Author affiliations}

${ }^{1}$ La Paz Central Research and Clinical Trials Unit, Hospital Universitario La Paz, Madrid, Spain

${ }^{2}$ Translational Research Unit in Paediatric Haemato-Oncology, Hematopoietic Stem Cell Transplantation and Cell Therapy, Hospital Universitario La Paz, Madrid, Spain ${ }^{3}$ Paediatric Haematology-Oncology Unit, Hospital Clínico Universitario Virgen de la Arrixaca, El Palmar, Spain

${ }^{4}$ Department of Paediatrics, Hospital Universitario Cruces, Barakaldo, Spain ${ }^{5}$ Paediatric Haematology Unit, Maternal and Children Hospital, Hospital Regional Universitario de Málaga, Málaga, Spain

${ }^{6}$ Paediatric Haematology Department, Maternal and Children Hospital, Complejo Hospitalario Universitario de Badajoz, Badajoz, Spain

${ }^{7}$ Haematological Malignancies Clinical Research Unit, Centro Nacional de Investigaciones Oncológicas (CNIO), Madrid, Spain

${ }^{8}$ Translational Research Unit in Paediatric Hemato-Oncology, Haematopoietic Stem Cell Transplantation and Cell Therapy, Institute of Medical and Molecular Genetics (INGEMM), Hospital Universitario La Paz, Madrid, Spain

${ }^{9}$ Paediatric Haemato-Oncology Deparment, Hospital Universitario La Paz, Madrid, Spain

${ }^{10}$ Clinical Pharmacology Department, Hospital Universitario La Paz, Madrid, Spain

Contributors APM and MVC conceived of the study. MMB, HYT and AMB provided statistical expertise in the clinical trial design and initiated the study design. JLFS, IA, APM, BGM, LS, MB and JMVV will help with clinical trial implementation and recruitment. JVQ, LFG, AEL, MVC and IMA will help with part of GMP manufacturing and laboratory experiments. IMA and MMB will help with regulatory aspects of advanced therapies. APM and MVC will conduct the primary statistical analysis. All authors contributed to refinement of the study protocol and approved the final manuscript.

Funding This study was supported in part by the National Health Service of Spain, Instituto de Salud Carlos III (ISCIII), FIS: PI15/00973, FONDOS FEDER, Mutua Madrileña Foundation and the CRIS Cancer Foundation (Project Lydia-2).

Competing interests None declared.

Patient consent for publication Not required.

Provenance and peer review Not commissioned; externally peer reviewed.

Data availability statement Data are available on reasonable request.

Open access This is an open access article distributed in accordance with the Creative Commons Attribution Non Commercial (CC BY-NC 4.0) license, which permits others to distribute, remix, adapt, build upon this work non-commercially, and license their derivative works on different terms, provided the original work is properly cited, appropriate credit is given, any changes made indicated, and the use is non-commercial. See: http://creativecommons.org/licenses/by-nc/4.0/.

\section{ORCID iDs}

Mario Muñoz Builes http://orcid.org/0000-0002-6436-9195

Miguel Blanquer http://orcid.org/0000-0002-1471-8828

\section{REFERENCES}

1 Rubnitz JE, Inaba H, Leung WH, et al. Definition of cure in childhood acute myeloid leukemia. Cancer 2014;120:2490-6.

2 Goswami M, Hourigan CS. Novel antigen targets for immunotherapy of acute myeloid leukemia. Curr Drug Targets 2017;18:296-303.
3 Pui C-H, Carroll WL, Meshinchi S, et al. Biology, risk stratification, and therapy of pediatric acute leukemias: an update. J Clin Oncol 2011;29:551-65.

4 Bolouri H, Farrar JE, Triche T, et al. The molecular landscape of pediatric acute myeloid leukemia reveals recurrent structural alterations and age-specific mutational interactions. Nat Med 2018;24:103-12.

5 Rubnitz JE, Inaba H, Ribeiro RC, et al. NKAML: a pilot study to determine the safety and feasibility of haploidentical natural killer cell transplantation in childhood acute myeloid leukemia. J Clin Oncol 2010;28:955-9.

6 Mailloux AW, Epling-Burnette PK. Effector memory regulatory T-cell expansion marks a pivotal point of immune escape in myelodysplastic syndromes. Oncoimmunology 2013;2:e22654.

7 Steger B, Milosevic S, Doessinger G, et al. CD4(+)and CD8(+)T-cell reactions against leukemia-associated- or minor-histocompatibilityantigens in AML-patients after allogeneic SCT. Immunobiology 2014;219:247-60.

8 Ruggeri L, Capanni M, Urbani E, et al. Effectiveness of donor natural killer cell alloreactivity in mismatched hematopoietic transplants. Science 2002;295:2097-100.

9 Farag SS, Fehniger TA, Ruggeri L, et al. Natural killer cell receptors: new biology and insights into the graft-versus-leukemia effect. Blood 2002;100:1935-47.

10 Yoon SR, Kim T-D, Choi I. Understanding of molecular mechanisms in natural killer cell therapy. Exp Mol Med 2015;47:e141-11.

11 Siegler U, Meyer-Monard S, Jörger S, et al. Good manufacturing practice-compliant cell sorting and large-scale expansion of single KIR-positive alloreactive human natural killer cells for multiple infusions to leukemia patients. Cytotherapy 2010;12:750-63.

12 Koehl U, Brehm C, Huenecke S, et al. Clinical grade purification and expansion of NK cell products for an optimized manufacturing protocol. Front Oncol 2013;3:1-12.

13 Velardi A, Ruggeri L, Mancusi A. Killer-cell immunoglobulin-like receptors reactivity and outcome of stem cell transplant. Curr Opin Hematol 2012;19:319-23.

14 Stringaris K, Sekine T, Khoder A, et al. Leukemia-induced phenotypic and functional defects in natural killer cells predict failure to achieve remission in acute myeloid leukemia. Haematologica 2014;99:836-47.

15 Leung W. Infusions of allogeneic natural killer cells as cancer therapy. Clin Cancer Res 2014;20:3390-400.

16 Nguyen R, Wu H, Pounds S, et al. A phase II clinical trial of adoptive transfer of haploidentical natural killer cells for consolidation therapy of pediatric acute myeloid leukemia. J Immunother Cancer 2019;7:81.

17 Fernández L, Leivas A, Valentín J, et al. How do we manufacture clinical-grade interleukin-15-stimulated natural killer cell products for cancer treatment? Transfusion 2018:58:1340-7.

18 Vela M, Corral D, Carrasco P, et al. Haploidentical IL-15/41BBL activated and expanded natural killer cell infusion therapy after salvage chemotherapy in children with relapsed and refractory leukemia. Cancer Lett 2018;422:107-17.

19 Robertson MJ, Cameron C, Lazo S, et al. Costimulation of human natural killer cell proliferation: role of accessory cytokines and cell contact-dependent signals. Nat Immun 1996-1997;15:213-26.

20 Lapteva N, Durett AG, Sun J, et al. Large-scale ex vivo expansion and characterization of natural killer cells for clinical applications. Cytotherapy 2012;14:1131-43.

21 Voskens CJ, Watanabe R, Rollins S, et al. Ex-vivo expanded human NK cells express activating receptors that mediate cytotoxicity of allogeneic and autologous cancer cell lines by direct recognition and antibody directed cellular cytotoxicity. J Exp Clin Cancer Res 2010;29:134.

22 Fujisaki $\mathrm{H}$, Kakuda $\mathrm{H}$, Shimasaki $\mathrm{N}$, et al. Expansion of highly cytotoxic human natural killer cells for cancer cell therapy. Cancer Res 2009;69:4010-7.

23 Leivas A, Pérez-Martínez A, Blanchard MJ, et al. Novel treatment strategy with autologous activated and expanded natural killer cells plus anti-myeloma drugs for multiple myeloma. Oncoimmunology 2016;5:e1250051.

24 González B, Bueno D, Rubio PM, et al. Aspectos inmunológicos de la leucemia mieloblástica aguda. Anales de Pediatría 2016:84:195-202.

25 Miller JS, Soignier Y, Panoskaltsis-Mortari A, et al. Successful adoptive transfer and in vivo expansion of human haploidentical NK cells in patients with cancer. Blood 2005;105:3051-7.

26 Björklund AT, Carlsten M, Sohlberg E, et al. Complete remission with reduction of high-risk clones following haploidentical NK-cell therapy against MDS and AML. Clin Cancer Res 2018;24:1834-44. 